\title{
On the Nature of the Compact Dark Mass at the Galactic Center
}

\section{Citation}

Broderick, Avery E., and Ramesh Narayan. 2006. "On the Nature of the Compact Dark Mass at the Galactic Center." The Astrophysical Journal 638 (1): L21-24. https://doi.org/10.1086/500930.

\section{Permanent link}

http://nrs.harvard.edu/urn-3:HUL.InstRepos:41384970

\section{Terms of Use}

This article was downloaded from Harvard University's DASH repository, and is made available under the terms and conditions applicable to Other Posted Material, as set forth at http:// nrs.harvard.edu/urn-3:HUL.InstRepos:dash.current.terms-of-use\#LAA

\section{Share Your Story}

The Harvard community has made this article openly available.

Please share how this access benefits you. Submit a story.

\section{Accessibility}




\title{
On The Nature of the Compact Dark Mass at the Galactic Center
}

\author{
Avery E. Broderick ${ }^{1}$ and Ramesh Narayan ${ }^{2}$ \\ Institute for Theory and Computation, Harvard-Smithsonian Center for Astrophysics, MS 51, 60 Garden \\ Street, Cambridge, MA 02138, USA
}

\begin{abstract}
We consider a model in which Sgr $\mathrm{A}^{*}$, the $3.7 \times 10^{6} M_{\odot}$ supermassive black hole candidate at the Galactic Center, is a compact object with a thermally emitting surface. For very compact surfaces within the photon orbit, the thermal assumption is likely to be a good approximation because of the large number of rays which are strongly gravitationally lensed back onto the surface. Given the very low quiescent luminosity of Sgr A* in the near infrared, the existence of a hard surface, even in the limit in which the radius approaches the horizon, places a severe constraint upon the steady mass accretion rate onto the source: $\dot{M} \lesssim 10^{-12} M_{\odot} \mathrm{yr}^{-1}$. This limit is well below the minimum accretion rate needed to power the observed submillimeter luminosity of Sgr A*: $\dot{M}>10^{-10} M_{\odot} \mathrm{yr}^{-1}$. We thus argue that Sgr A* does not have a surface, i.e., it must have an event horizon. The argument could be made more restrictive by an order of magnitude with $\mu$ as resolution imaging, e.g., with submillimeter very-long baseline interferometry.
\end{abstract}

Subject headings: Galaxy: center-submillimeter-infrared: general—black hole physics-accretion, accretion disks - gravitational lensing

\section{Introduction}

Infrared observations of individual stars in the Galactic Center imply the existence of a dark object of mass $M \approx 3.7 \times 10^{6} M_{\odot}$, constrained to lie within $45 \mathrm{AU}$ (or $10^{3} G M / c^{2}$ ) of the radio source Sgr A* (Schödel et al. 2003; Ghez et al. 2005a; Eisenhauer et al. 2005). Observations at $3.5 \mathrm{~mm}$ and $7 \mathrm{~mm}$ further constrain the extent of the radio emission from Sgr A* to less than $1-2 \mathrm{AU}$ (or $10-20 G M / c^{2}$ ) (Shen et al. 2005; Bower et al. 2004). The favored interpretation of these observations is that $\mathrm{Sgr} \mathrm{A}^{*}$ is a supermassive black hole. Indeed, the current constraints rule out many alternative explanations, including clusters of stellar mass compact objects (Maoz 1998) and fermion balls (Schödel et al. 2002). Nonetheless, it remains to be conclusively demonstrated that the dark mass at the Galactic Center is a true black hole with an event horizon.

If Sgr $\mathrm{A}^{*}$ is not a black hole, then it must have

\footnotetext{
1 abroderick@cfa.harvard.edu

${ }^{2}$ rnarayan@cfa.harvard.edu
}

a surface at some radius $R$. Although general relativistic considerations coupled with reasonable assumptions on the equation of state of matter require $R \geq 9 G M / 4 c^{2}$ (see, e.g., Shapiro \& Teukolsky 1986), alternatives to general relativity exist which allow smaller radii, despite the fact that the exterior spacetime may be arbitrarily close to that predicted by general relativity (e.g., scalar-tensor theories, Fujii \& Maeda 2003, gravastars, Mazur \& Mottola 2001, boson stars Torres et al. 2000). Thus, in principle, $R$ could have any value greater than $2 G M / c^{2}$, the horizon radius (we restrict our analysis to non-spinning objects). In this Letter, we show that current observations do not favor Sgr A* having such a surface.

We assume that any putative surface of Sgr A* is in steady state in the presence of accreting gas, and that it emits the accreted energy thermally. The latter assumption is reasonable since, even for models in which the radius of the surface approaches $2 G M / c^{2}$, the thermalization timescale is short in comparison to the lifetime of Sgr A* (or of 
an observer $)^{1}$. Indeed, for surfaces contained well within the photon orbit, strong gravitational lensing significantly decreases the number of photon trajectories that escape to infinity. Most outgoing rays return to be absorbed by other parts of the surface, so that the object will resemble the classical example of a blackbody: a thermal cavity with a pinhole.

If $\operatorname{Sgr} \mathrm{A}^{*}$ accretes at the Bondi rate from the hot gas surrounding it, the accretion rate is expected to be $\dot{M} \sim 10^{-6} M_{\odot} \mathrm{yr}^{-1}$ (Baganoff et al. 2003). A more likely scenario is that the source accretes via a radiatively inefficient accretion flow (RIAF; Narayan et al. 1995; Yuan et al. 2003, and references therein), with a mass accretion rate in the range $\dot{M} \sim 10^{-8.5}-10^{-6} M_{\odot} \mathrm{yr}^{-1}$. In a RIAF model, essentially all the potential energy released by the accreting gas would be radiated from the surface of Sgr A* (assuming it has a surface), with a predicted luminosity at infinity of

$$
L_{\mathrm{surf}} \approx \eta \dot{M} c^{2}
$$

where the efficiency factor $\eta$ is the fraction of the rest mass energy of the infalling gas that is released as radiation. In the Newtonian case, $\eta$ is simply $G M / c^{2} R$. In general relativity, $\eta$ is given in terms of the gravitational redshift $z$ at the surface:

$$
\eta=z /(1+z), \quad 1+z=\left(1-2 G M / c^{2} R\right)^{-1 / 2} .
$$

Although it is highly unlikely that $\operatorname{Sgr} \mathrm{A}^{*}$ has a radiatively efficient accretion disk, even such a model requires a fairly large $\dot{M}$. For example, the observed bolometric luminosity of Sgr A* of $10^{36} \mathrm{erg} \mathrm{s}^{-1}$ implies a minimum accretion rate of $\dot{M} \sim 2 \times 10^{-10} M_{\odot} \mathrm{yr}^{-1}$ for a radiative efficiency of $10 \%$. To within a factor of a few (depending on the nature of the boundary layer at the inner edge of the disk), the luminosity from the surface of Sgr $\mathrm{A}^{*}$ is again predicted to be $\sim \eta \dot{M} c^{2}$ with $\eta$ not very different from (2). All the above estimates are for gas accretion. When stellar capture events are considered, the average accretion rate can be as high as $10^{-5} M_{\odot} \mathrm{yr}^{-1}$ to $10^{-3} M_{\odot} \mathrm{yr}^{-1}$,

\footnotetext{
${ }^{1}$ The thermalization timescale is expected to be on the order of the cooling time of the infalling matter, which is not very different from the free-fall time-scale $\sim 100 \mathrm{~s}$ at the surface. Since the timescale diverges only logarithmically as measured at infinity even for extremely compact configurations (e.g., the gravastar), the thermalization time is increased by only a few orders of magnitude.
}

though this would be expected to be in the form of transient accretion events (Magorrian \& Tremaine 1999). Note that the $\dot{M}$ estimates given here are from the point of view of a distant observer, i.e., they represent the rate of accretion of rest mass per unit time as measured at infinity.

In $\oint 2$, we derive upper limits on $\dot{M}$ from the observed near-infrared (NIR) fluxes of Sgr A* and compare these with the various estimates of $\dot{M}$ given above. On this basis we argue that Sgr $\mathrm{A}^{*}$ is unlikely to have a surface and therefore that it must be a black hole. In $\S 3$, we present theoretical images of the RIAF model discussed in Yuan et al. (2003) and Broderick \& Loeb (2005a), and show that imaging experiments alone cannot distinguish between a black hole and a compact object with a surface. However, by combining imaging with the argument presented in $\S 2$, we show that one could make the case for an event horizon stronger. We conclude in $\S 4$ with a discussion. In what follows, unless otherwise noted, we use geometrized units $(G=c=1)$.

\section{NIR Limits on the Mass Accretion Rate}

The observed flux $F_{\nu}$ at a frequency $\nu$ from a surface emitting thermal blackbody radiation is simply the blackbody spectrum multiplied by the apparent solid angle of the surface on the sky. For a thermally emitting compact spherical surface at the Galactic Center this is

$$
F_{\nu}=\frac{2 h \nu^{3}}{c^{2}} \frac{\mathrm{e}^{-h \nu / k T}}{1-e^{-h \nu / k T}} \frac{\pi b^{2}}{D^{2}},
$$

where $T$ is the blackbody temperature as measured by the observer (at infinity), $D \simeq 8 \mathrm{kpc}$ is the distance to the Galactic Center, and the apparent size $b$ of the radiating surface is given in terms of its radius $R$ by

$$
b^{2}=\left\{\begin{aligned}
27 M^{2}, & R<3 M \\
R^{3} /(R-2 M), & R \geq 3 M
\end{aligned}\right.
$$

Equation (4) includes the general relativistic correction due to strong lensing. Note that, for $R$ inside the photon orbit $3 M$, the apparent size $b$ no longer decreases with decreasing $R$.

For a given choice of radius $R$ and a given upper limit on the flux $F_{\nu}$, equations (3) and (4) provide an upper limit $T_{\max }$ on the observed temperature 


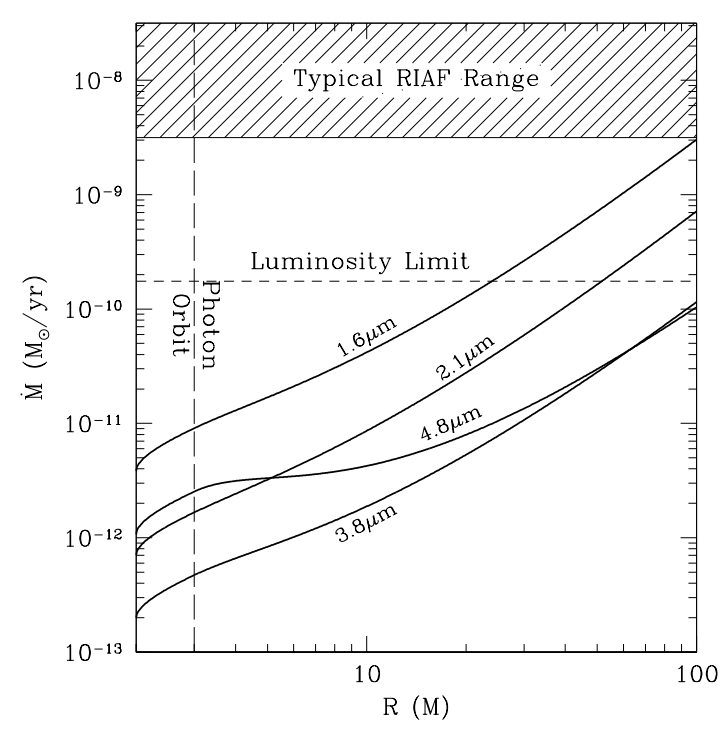

Fig. 1.- The four curves show upper limits on the mass accretion rate of $\mathrm{Sgr} \mathrm{A} \mathrm{A}^{*}$ as a function of the surface radius $R$, derived from the observed quiescent fluxes at 1.6, 2.1, 3.8 and $4.8 \mu \mathrm{m}$ listed in Table 1. The surface is assumed to radiate as a blackbody. For reference, the photon orbit $R=3 M$ is shown by the vertical dashed line. The cross-hatched area at the top corresponds to typical mass accretion rates in RIAF models of Sgr $\mathrm{A}^{*}$, and the horizontal dashed line represents the minimum accretion rate needed to power the bolometric luminosity of Sgr A*(see $\S 1)$.

(at infinity) and hence a limit on the surface luminosity as measured at infinity: $L_{\text {surf }}<4 \pi b^{2} \sigma T_{\max }^{4}$. Then, from equations (1) and (2) we obtain the following upper limit on the mass accretion rate on the surface,

$$
\dot{M}<4 \pi b^{2} \sigma T_{\max }^{4} / c^{2} \eta .
$$

Thus, for each flux measurement $F_{\nu}$ of Sgr A* and an assumed radius $R$, we obtain an estimate of $\dot{M}_{\max }(R)$, the maximum accretion rate for that $R$. This limit, which assumes nothing more than blackbody emission, can be compared with the mass accretion rates which are required to explain the observed luminosity and spectra of Sgr A* (discussed in $\S 1$ ).

In practice, the strongest limits are placed by observations in the NIR, since the postulated ther- mal emission from the surface of Sgr A* peaks in this region of the spectrum. The most constraining observations are listed in Table 1, and the corresponding limits on $\dot{M}(R)$ are plotted in Figure 1. Note that the thermal emission from the surface peaks in the NIR for $R \sim 10 M$, whereas for larger radii the surface is sufficiently cool that the observed fluxes fall in the Wien regime, resulting in a weakening of the limit on $T_{\max }$ and hence on $\dot{M}$. The difference in character between the limit imposed by observations at $4.8 \mu \mathrm{m}$ and the other wavelengths is due to the fact that the former passes into the Rayleigh-Jeans regime at a larger radius. The overall limit on $\dot{M}$ is simply given by the lower envelope of all four curves in Figure 1; the envelope is dominated by the $3.8 \mu \mathrm{m}$ curve.

Recent observations at $3.5 \mathrm{~mm}$ and $7 \mathrm{~mm}$ have claimed to resolve Sgr $\mathrm{A}^{*}$, limiting the radius to $R \lesssim 10-20 M$ at these wavelengths (Shen et al. 2005; Bower et al. 2004). As seen in Figure 1 , this limit on the radius, coupled with the NIR flux limits, already restricts the allowed accretion rate in $\mathrm{Sgr} \mathrm{A}^{*}$ to $\dot{M} \lesssim 10^{-12} M_{\odot} / \mathrm{yr}$, if the object has a surface. Since the derived limit is two orders of magnitude lower than the lowest $\dot{M}$ allowed by the observed luminosity of Sgr $\mathrm{A}^{*}\left(\sim 2 \times 10^{-10} M_{\odot} \mathrm{yr}^{-1}\right)$, and more than three orders of magnitude lower than the lowest accretion rate required by RIAF models (see $\S 1$ ), the case for Sgr $A^{*}$ not having a surface, i.e., for it being a black hole, is very strong. As is apparent from Figure 1, observations which can further limit $R$ will place even stronger constraints upon $\dot{M}$, and further strengthen the case for a black hole.

\section{Images}

Submillimeter very-long baseline interferometry (VLBI) promises to provide an angular resolution on the order of $\sim 20 \mu \mathrm{as}$, corresponding to a physical scale $\sim 5 M$ at the Galactic Center. A significant motivation for developing such a capability is the prospect of imaging the silhouette or "shadow" of the central black hole (Falcke et al. 2000; Miyoshi et al. 2004; Doeleman \& Bower 2004; Broderick \& Loeb 2005a,b,c). The shadow is due to strong lensing, and its detection would be a strong confirmation of one of the major predictions of general relativity in the limit of strong 
TABLE 1

Near-Infrared Flux Limits of SGR A*

\begin{tabular}{ccc}
\hline \hline$\lambda(\mu \mathrm{m})$ & $F_{\nu}(\mathrm{mJy})$ & Ref. \\
\hline 1.6 & 11 & Stolovy et al. (2003) \\
2.1 & 2.8 & Ghez et al. (2005b) \\
3.8 & 1.28 & Ghez et al. (2005b) \\
4.8 & 3.5 & Clénet et al. (2004) \\
\hline
\end{tabular}

\section{Black Hole}

$\mathrm{R}=2.02 \mathrm{M}$

$9 \mathrm{M} / 4$
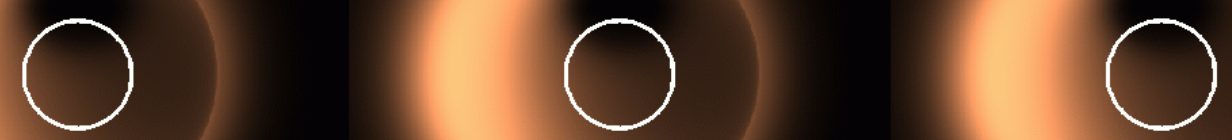

$3 \mathrm{M}$

$4 \mathrm{M}$

$6 \mathrm{M}$
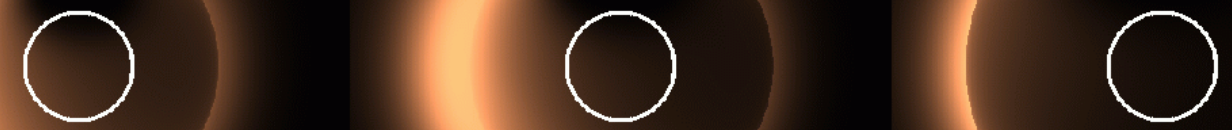

Fig. 2. - $350 \mathrm{GHz}$ images of a RIAF model of $\operatorname{Sgr} \mathrm{A}^{*}$ (the $a=0$ model in Broderick \& Loeb 2005a) in which the gas accretes onto a compact thermally emitting surface in a Schwarzschild spacetime. The panels correspond to different assumed radii of Sgr $\mathrm{A}^{*}$, given in the upper left corner of each image, ranging from $1 \%$ larger than the horizon to $6 M$. For comparison, an image of the same accretion flow onto a black hole is shown in the upper-left panel. All the images correspond to a viewing angle $45^{\circ}$ above the orbital plane. The brightness scale is normalized separately in each image, ranging from maximum intensity to zero (black). The white circles show the size of the Schwarzschild horizon $2 M$. Note that the thermally emitting surface is practically invisible. This is because of its very low temperature $\left(\sim 10^{4} \mathrm{~K}\right)$ compared to the brightness temperature of the relativistic accreting gas $\left(>10^{10} \mathrm{~K}\right)$. 
gravity. Such imaging will also constrain the radius of $\operatorname{Sgr} A^{*}$ 's surface and thus strengthen the argument presented in $\S 2$.

Figure 2 shows theoretical submillimeter VLBI images of a RIAF model of Sgr $A^{*}$ for a number of assumed radii ranging from $1 \%$ larger than the horizon to $6 M$. In all cases the mass accretion rate is taken to be $10^{-8} M_{\odot} / \mathrm{yr}$, the canonical value for RIAF models and orders of magnitude above the limits placed in the preceding section. Surface radii in excess of the photon orbit $(3 M)$ appear as enlarged silhouettes, as may be seen in the bottom panels. Thus, high resolution imaging can immediately limit the size of any radiating surface in Sgr A* to less than $3 M$. Combined with the NIR flux limits, this would strengthen the limit upon the mass accretion rate by nearly another order of magnitude, making a conclusive case for an event horizon.

Interestingly, we see that for radii inside of the photon orbit, the images are nearly indistinguishable from the case in which a horizon is present. This is a direct result of the relatively low temperature of the radiating surface $\left(\sim 10^{4} \mathrm{~K}\right.$ as measured at infinity) coupled with the fact that at submillimeter wavelengths the emission occurs deep in the Rayleigh-Jeans portion of the spectrum. Thus, while submillimeter imaging can limit the radius of Sgr $A^{*}$, it cannot by itself prove that the object is a black hole. It is only when the imaging results are combined with the NIR flux measurements in the manner discussed in $\S 2$ that a strong case can be made for the existence of a horizon.

\section{Discussion}

We have shown in this Letter that current NIR flux limits on Sgr A* already place a stringent upper limit upon the mass accretion rate of this compact object, assuming that the object possesses a surface. Comparison of these rates to the observed submillimeter luminosity of the source, and the implied lower limit on the mass accretion rate, leads to a serious contradiction, thus providing strong evidence for the absence of a surface. This argument for Sgr A* being a black hole is robust (see Narayan et al. 1997, 2002; Narayan \& Heyl 2002, and references therein, for other evidence). The argument applies even when the surface is extremely compact, e.g., as expected in gravastar models (see, e.g., Mazur \& Mottola 2001). Note from equations (1) and (2) that the extremely large gravitational redshift at the surface of an ultra-compact object $(R \rightarrow 2 M)$ does not cause a reduction in the luminosity observed at infinity. On the contrary, for a given $\dot{M}$, the observed luminosity is maximum when the redshift goes to infinity (this is in contrast to Abramowicz et al. 2002, in which the intrinsic luminosity measured on the surface, as opposed to the mass accretion rate measured at infinity, was assumed to be fixed). In addition, the blackbody assumption on which our argument is based is particularly well motivated when the redshift is large $(\S 1)$.

Three critical assumptions underly our conclusions: $(i)$ the surface is in steady state with respect to the accreting material, $(i i)$ the surface radiates thermally, and ( iii) general relativity is an appropriate description of gravity external to the surface.

As mentioned briefly in $\S 1$, the assumption of steady state is likely to be a good one, even for surfaces very near the horizon (including those which are separated from the horizon by a Planck length, the minimum scale for which a horizon will not develop). However, it should be noted that for a black hole the unradiated binding energy of the accreting matter contributes to an increase of the black hole's mass. Thus a black hole is an explicit example of an accreting compact object which is not in steady state.

Of more concern is the assumption that the surface emits thermally. For models in which largescale correlations play a significant role (e.g., the gravastar) it is unclear what happens to accreting material. For instance, it is conceivable that one may obtain coherent emission with wavelengths comparable to the correlation length of the surface, which in principle could introduce large deviations from the Planck spectrum. While we cannot rule out such a model, we would like to emphasize that, in general, for the gravastar (or similar) model to remain a viable alternative to the black hole model of Sgr A* necessarily requires an extremely exotic emission mechanism that deviates enormously from blackbody emission.

Finally, some assumption regarding the description of gravity external to the surface is necessary to compute the flux due to a compact surface near a strongly gravitating object. In the absence of 
a well tested alternative, general relativity is the natural choice and this is what we have selected for our calculations. While we have explicitly considered a non-rotating black hole, we expect rotation (the most obvious extension that one would wish to consider) to primarily broaden the thermal spectrum, changing our results by no more than factors of order unity. Multi-wavelength highresolution imaging of flares in the Galactic Center has been proposed as a method by which the nature of the spacetime surrounding the Galactic Center's black hole may be probed (Broderick \& Loeb 2005b,c).

To summarize, in the absence of unknown exotic phenomena, the current NIR flux measurements already conclusively imply the existence of an event horizon in the black hole candidate Sgr $\mathrm{A}^{*}$ at the Galactic Center.

We would like to thank Avi Loeb for pointing out the highly enhanced accretion rate of Sgr A* due to stellar capture events. This work was supported in part by NSF grant AST 0307433. A.E.B. gratefully acknowledges the support of an ITC Fellowship from Harvard College Observatory.

\section{REFERENCES}

Abramowicz, M. A., Kluźniak, W., \& Lasota, J.-P. 2002, A\&A, 396, L31

Baganoff, F. K., Maeda, Y., Morris, M., et al. 2003, ApJ, 591, 891

Bower, G. C. et al. 2004, Science, 304, 704

Broderick, A. E. \& Loeb, A. 2005a, ApJL, submitted (astro-ph/0508386)

Broderick, A. E. \& Loeb, A. 2005b, MNRAS, 363, 353

Broderick, A. E. \& Loeb, A. 2005c, MNRAS, submitted (astro-ph/0509237)

Clénet, Y. et al. 2004, A\&A, 424, L21

Doeleman, S. \& Bower, G. 2004, Galactic Center Newsletter, 18, 6

Eisenhauer, F. et al. 2005, ApJ, 628, 246

Falcke, H., Melia, F., \& Agol, E. 2000, ApJL, 528, L13
Fujii, Y. \& Maeda, K.-I. 2003, The Scalar-Tensor Theory of Gravitation (Cambridge : Cambridge University Press, 2003.)

Ghez, A. M. et al. 2005a, ApJ, 620, 744

Ghez, A. M. et al. 2005b, astro-ph/0508664

Magorrian, J. \& Tremaine, S. 1999, MNRAS, 309, 447

Maoz, E. 1998, ApJL, 494, L181

Mazur, P. O. \& Mottola, E. 2001, gr-qc/0109035

Miyoshi, M., Ishitsuka, J. K., Kameno, S., Shen, Z., \& Horiuchi, S. 2004, Progress of Theoretical Physics Supplement, 155, 186

Narayan, R., Garcia, M. R., \& McClintock, J. E. 1997, ApJL, 478, L79

Narayan, R., Garcia, M. R., \& McClintock, J. E. 2002, in The Ninth Marcel Grossmann Meeting, $405-425$

Narayan, R. \& Heyl, J. S. 2002, ApJL, 574, L139

Narayan, R., Yi, I., \& Mahadevan, R. 1995, Nature, 374,623

Schödel, R. et al. 2002, Nature, 419, 694

Schödel, R. et al. 2003, ApJ, 596, 1015

Shapiro, S. L. \& Teukolsky, S. A. 1986, Black Holes, White Dwarfs and Neutron Stars: The Physics of Compact Objects (New York : Wiley-VCH , 1986.)

Shen, Z.-Q. et al. 2005, Nature, 000, 000

Stolovy, S., Melia, F., McCarthy, D., \& YusefZadeh, F. 2003, Astronomische Nachrichten Supplement, 324, 419

Torres, D. F., Capozziello, S., \& Lambiase, G. 2000, Phys. Rev. D, 62, 104012

Yuan, F., Quataert, E., \& Narayan, R. 2003, ApJ, 598,301

This 2-column preprint was prepared with the AAS LATEX macros v5.2. 